\title{
A checklist of the freshwater rotifer fauna of Thailand (Rotifera, Monogononta, Bdelloidea)
}

\author{
Phannee SA-ARDRIT, ${ }^{1,2}$ Pornsilp PHOLPUNTHIN, ${ }^{1}$ Hendrik SEGERS ${ }^{3 *}$ \\ ${ }^{1}$ Department of Biology, Prince of Songkla University, 90112 Hat Yai; ${ }^{2}$ Princess Maha Chakri Sirindhorn Natural History Museum, \\ Prince of Songkla University, 90112 Hat Yai, Thailand; ${ }^{3}$ Royal Belgian Institute of Natural Sciences, Vautierstraat 29, 1000 Brussels, \\ Belgium \\ *Corresponding author: hsegers@naturalsciences.be
}

\begin{abstract}
We provide a checklist of the freshwater Rotifera recorded from Thailand, based on a review of available literature. Approximately 398 species of rotifers are recorded. The rotifer faun of Thailand has been investigated quite comprehensively and these studies focus on four main parts of the country: the northern, north-eastern, central and southern. The number of rotifers on record is the highest in the north-eastern part (275), followed by the southern part (261), the central part (182) and the northern part (115). The majority of Thai Rotifera belongs to family Lecanidae (24.4\%), Lepadellidae (11.3\%), Brachionidae (11.1\%), Trichocercidae (9.0\%) and Flosculariidae (9.0\%). The most diverse genus is Lecane followed by Trichocerca, Lepadella and Brachionus. Although most Thai rotifers are cosmopolitan, there are a number of Oriental endemics, including some strict Thai or regional endemics. Illoricate rotifers and bdelloids are understudied, while experimental and molecular approaches promise to add most to our knowledge and understanding of the role of rotifers in ecosystem functioning.
\end{abstract}

Key words: Rotifer, biodiversity, biogeography, endemicity, Thailand.

Received: February 2013. Accepted: June 2013.

\section{INTRODUCTION}

Phylum Rotifera is, together with Cladocera and Copepoda, one of three main groups of freshwater zooplankton. In many cases, it contributes the highest number of species to local zooplankton diversity. As with many taxonomic groups, our perception of its diversity has evolved over the years as a consequence of advances in taxonomic knowledge and the availability of new study techniques. This has resulted in the presence of a number of ambiguous records in the literature. In particular, in Thailand rotifers have been recorded over a time span of over a century, starting by Weber (1907), and quite a few older records refer to taxonomic concepts that are now understood differently than at the time they were published, and/or use names that are no longer considered valid.

The application of molecular tools promises to revolutionise our understanding of species-level diversity of many organisms, and this is not different in Rotifera (e.g. Fontaneto et al., 2009; García-Morales and Elías-Gutiérrez, 2013; Leasi et al., 2013). Considering this, we feel that the time has come to re-evaluate available literature records of Thai Rotifera, to serve as a basis for future work utilising the new techniques that are increasingly becoming available. We therefore propose the first checklist of the Rotifera of Thailand, taking into account the most recent insights in the taxonomy and biogeography of the animals, aligned with contemporary views on taxonomy, diversity and biogeography of Rotifera (Segers 2007, 2008; Segers and De Smet, 2008).

\section{METHODS}

Our checklist contains the valid names of genera and species recorded from Thailand, and includes synonyms listed in the relevant literature starting by Weber (1907) and including Meksuwan et al. (2011), the most recent paper known to us. Unless stated otherwise, taxonomy and nomenclature follow Segers (2007). We further list infrasubspecific variants recorded under their nominal species, as appropriate. Occurrence of species is indicated by region as defined by Setapan (1999), who recognises six geographical regions (northern, northeastern, central, western, eastern and southern) defined by mountain ranges and topography in Thailand. For the biogeographical comparison we refer to regions as defined in Balian et al. (2008). Information on the distribution of rotifer species is obtained from Segers (1996, 2007), except for those species-level taxa that were, for any reason, not included in those works [Collotheca algicola (Hudson, 1886), Lepadella monostyla f. caudata (Koste, 1972), Stephanoceros millsii (Kellicott, 1885), Trichocerca inermis (Linder, 1904) and Trichocerca tenuidens (Hauer, 1931)]. 


\section{RESULTS AND DISCUSSION}

The study of freshwater rotifers in Thailand started with the publication by Weber (1907). To date, approximately 398 species-level taxa of rotifer have been recorded (Tab. 1). A number of older records were recently re-evaluated by Segers and Savatenalinton (2010). Other dubious or otherwise problematic records are the following:

- Brachionus dichotomus Shephard, 1911 and B. dichotomus reductus Koste and Shiel, 1980. Both names can be found in literature dealing with Thai Rotifera; however, the typical B. dichotomus has, to our knowledge, not been recorded from the Oriental region and we therefore indicate that the presence of $B$. dichotomus Shephard requires confirmation.

- Brachionus plicatilis Müller, 1786: records under this name most likely do not refer to this species as presently understood (Ciros-Perez et al., 2001), but any of the warm-water representatives of this cryptic species group (Suatoni et al., 2006).

- Keratella tropica Apstein, 1907: we included under this name the record of Keratella valga (Ehrenberg, 1834) by Boonsom (1984), considering that the distinction between the cold-water, acidophilic $K$. valga and the warm-water, euryoecious $K$. tropica has long remained problematic, and that it is unlikely that the former would occur in the habitats studied by Boonsom (1984).

- Koste's (1975) record of the nomen nudum Lepadella monostyla f. caudata (Koste) is most likely a lapsus regarding the taxon Lepadella monodactyla caudata Koste, 1972, at present considered a junior subjective synonym of the nominal taxon.

- The presence in Thailand of Filinia terminalis (Plate, 1886) is in need of confirmation, considering that this cold-stenotherm is easily confused with the warmwater F. novaezealandiae Shiel and Sanoamuang, 1993 (Segers et al. 1996). However, while the two records of $F$. terminalis indeed precede the recognition of $F$. novaezealandiae, the presence of this species in the North of Thailand can not be excluded a priori.

- The record of Habrotrocha recumbens Bartoš, 1963 by Koste (1975) is not included in the checklist. The name is unavailable in the sense of the International Code of Zoological Nomenclature (ICZN, 1999), as it is based solely on the case inhabited by the animal, and not the animal itself.

- Of the remaining species not included in Segers (2007), Collotheca algicola (Hudson, 1886) and Stephanoceros millsii (Kellicott, 1885) were recently treated as valid by Meksuwan et al. (2011), while Trichocerca inermis (Linder, 1904) has recently been recognised as a junior synonym of $T$. dixonnuttalli (Jennings, 1903) (Jersabek et al., 2011), and Trichocerca tenuidens (Hauer, 1931) remains an insufficiently described species inquirenda (Segers, 2003).
The majority of Thai Rotifera belongs to family Lecanidae (1 genus with 97 species, 24.4\%), Lepadellidae (4 genera with 45 species, $11.3 \%$ ), Brachionidae (5 genera with 44 species, $11.1 \%$ ), Trichocercidae (1 genus with 36 species, 9.0\%) and Flosculariidae (9 genera with 36 species, 9.0\%). The most diverse genus was Lecane, comprising 97 species, followed by Trichocerca (36 species), Lepadella (31 species) and Brachionus (30 species).

Regarding the regional distribution of rotifers in the six geographical regions of Thailand (northern, north-eastern, central, western, eastern and southern) (Setapan, 1999), we note that Thailand has been quite comprehensively investigated, but studies have focused on four main parts: the northern, the north-eastern, the central and the southern. The number of rotifers on record is highest in the northeastern part (275 species) (Sanoamuang et al., 1995; Sanoamuang and Savatenalinton, 1999, 2001; Segers et al., 2004; Savatenalinton and Segers, 2005), followed by the southern part (261 species) (Pholpunthin, 1997; Segers and Pholpunthin, 1997; Pholpunthin and Chittapun, 1998; Chittapun et al., 1999, 2002, 2003, 2005, 2007; Chittapun and Pholpunthin, 2001; Segers and Chittapun, 2001; Sanoamuang, 2007; Meksuwun et al., 2011), the central part (182 species) (De Ridder, 1971; Koste, 1975; Sanoamuang and Segers, 1997; Jithland and Wongrat, 2006; Teeramaethee et al., 2006; Savatenalinton and Segers, 2008; Chittapun et al., 2009; Chittapun, 2011) and the northern part (115 species) (De Ridder, 1971; Sanoamuang, 1998).

That the number of rotifer species recorded from north-east Thailand is the highest of all regions should not automatically be interpreted as indicating that rotifer diversity is the highest there. Admittedly, this region is the largest of all Thai regions, but it is also the most intensively studied region, as can be judged from the number and of the relevant studies and their being comprehensive (e.g. Sanoamuang et al., 1995). The southern region has the second largest rotifer record, which we attribute to the high diversity and abundance of its freshwater habitats, in addition to intensive study. The record from other Thai regions is much lower, mostly because the number of studies is, conversely, lower. Nevertheless, when compared to other countries in Southeast Asia, the inventory confirms that the country is the best documented of all countries in the region (Segers, 2001).

The Thai rotifer fauna is composed largely of widespread, cosmopolitan or tropicopolitan species, but there is a sizeable fraction of Oriental (13 species, 3.3\%) or even local, Thai endemics (13 species, 3.3\%; Tab. 2). It can be expected that the latter number will decrease as research in countries neighbouring Thailand may reveal the presence of these strict Thai endemics, as is the case with species like Cephalodella songkhlaensis Segers and Pholpunthin, 1997 and Ptygura thalenoiensis Meksuwan et al., 2011. 


\section{CONCLUSIONS}

Notwithstanding that the Thai rotifer fauna is the richest of all Southeast Asian countries, this by no means implies that the fauna is adequately studied. In addition to knowledge gaps concerning understudied regions in Thailand, some taxonomic groups are insufficiently documented. This is the case for taxa such as Notommatidae and bdelloids [respectively 24 and 4 Thai records compared to 48 and 58 from the Oriental region (Segers, 2008)], two groups which are notoriously difficult to study because of methodological constraints. Furthermore, we lack understanding of the ecology and evolution of rotifers, and their role in ecosystem processes (Wallace et al., 2006). Targeted efforts on understudied regions and taxonomic groups, preferably using modern, molecular tools, will undoubtedly raise the records of rotifer diversity in Thailand, while experimental studies are needed to elucidate their contribution to ecosystem processes and functions.

\section{ACKNOWLEDGMENTS}

This publication is based on work presented during a workshop on diversity and ecology of freshwater zooplankton in southeast Asia, Guangzhou, China, 2-7 July 2011. We acknowledge grants from the Royal Golden Jubilee PhD Program (No. 4.B.PS/52/D.2), the Faculty of Science, Department of Biology, Prince of Songkla University, and the Institute of Hydrobiology, Jinan University, Guangzhou (Prof. Dr. H. Boping) for the opportunity to attend the workshop. We are most grateful to the organisers of the FISA meeting, Prof. Dr. L. Sanoamuang and Dr. Maria K. Hołyńska, for supporting the participation of HS in the Conference and the opportunity to submit the present contribution to the Proceedings of the meeting.

Finally, we thank two anonymous reviewers for their valuable and constructive comments on this manuscript.

Tab. 1. Species list of Rotifera in Thailand.

\begin{tabular}{|c|c|c|c|}
\hline & Species & Distribution & References \\
\hline & \multicolumn{3}{|l|}{ Monogononta } \\
\hline \multicolumn{4}{|c|}{ Family Asplanchnidae } \\
\hline 1 & Asplanchna brightwellii Gosse, 1850 & $\mathrm{NE}, \mathrm{C}$ & $1,3,4,13,17,20,21,22$ \\
\hline 2 & Asplanchna priodonta Gosse, 1850 & $\mathrm{~N}, \mathrm{NE}, \mathrm{C}$ & $3,4,9,13,17,20,21,22,24$ \\
\hline 3 & Asplanchna sieboldii (Leydig, 1854) & $\mathrm{N}, \mathrm{NE}, \mathrm{C}, \mathrm{S}$ & $4,9,17,23,24,26,28$ \\
\hline 4 & Asplanchna tropica Koste \& Tobias, 1989 & $\mathrm{NE}, \mathrm{S}$ & $4,5,7$ \\
\hline 5 & Asplanchnopus hyalinus Harring, 1913 & $\mathrm{NE}$ & 4,17 \\
\hline 6 & Asplanchnopus multiceps (Schrank, 1793) & $\mathrm{NE}$ & 4 \\
\hline 7 & Harringia rousseleti de Beauchamp, 1912 & S & 23 \\
\hline \multicolumn{4}{|c|}{ Family Atrochidae } \\
\hline 8 & Acyclus inquietus Leidy, 1882 & S & 29 \\
\hline 9 & Cupelopagis vorax (Leidy, 1857) & $\mathrm{C}, \mathrm{S}$ & $2,5,7$ \\
\hline \multicolumn{4}{|c|}{ Family Brachionidae } \\
\hline 10 & Anuraeopsis coelata de Beauchamp, 1932 & $\mathrm{NE}, \mathrm{C}, \mathrm{S}$ & $4,5,7,17,18,20,21,22,23,24$ \\
\hline 11 & $\begin{array}{l}\text { Anuraeopsis fissa Gosse, } 1851 \\
\text { Syn.: Anuraeopsis fissa fissa Gosse, 1851: Koste, } 1975\end{array}$ & $\mathrm{~N}, \mathrm{NE}, \mathrm{C}, \mathrm{S}$ & $\begin{array}{l}1,2,3,4,5,7,9,10,12,13,15,17,20,21,22, \\
23,24,26\end{array}$ \\
\hline 12 & Anuraeopsis navicula Rousselet, 1911 & $\mathrm{NE}, \mathrm{C}, \mathrm{S}$ & $4,5,7,10,21,23,24,26$ \\
\hline 13 & $\begin{array}{l}\text { Brachionus angularis Gosse, } 1851 \\
\text { Incl. Brachionus angularis Gosse f. typica, f. chelonis: } \\
\text { Sanoamuang et al., } 1995\end{array}$ & $\mathrm{~N}, \mathrm{NE}, \mathrm{C}, \mathrm{S}$ & $\begin{array}{l}1,3,4,5,7,9,10,13,17,18,20,21,22,23,24 \\
25,26\end{array}$ \\
\hline 14 & Brachionus angularis bidens Plate, 1886 & $\mathrm{NE}$ & 4 \\
\hline 15 & Brachionus bennini Leissling, 1924 & $\mathrm{NE}$ & 4 \\
\hline 16 & Brachionus bidentatus Anderson, 1889 & $\mathrm{NE}, \mathrm{C}$ & $4,13,17,21,24$ \\
\hline 17 & Brachionus budapestinensis Daday, 1885 & $\mathrm{NE}$ & 4,24 \\
\hline 18 & Brachionus calyciflorus Pallas, 1766 & $\mathrm{~N}, \mathrm{NE}, \mathrm{C}, \mathrm{S}$ & $1,3,4,5,7,9,13,17,18,20,21,22,23,24,26$ \\
\hline
\end{tabular}


Tab. 1. Continued from previous page.

\begin{tabular}{|c|c|c|c|}
\hline & Species & Distribution & References \\
\hline 19 & $\begin{array}{l}\text { Brachionus caudatus } \text { Barrois and Daday, } 1894 \\
\text { Incl. Brachionus caudatus f. aculeatus: Sanoamuang et al., 1995; } \\
\text { Teeramaethee et al., } 2006 \\
\text { Brachionus caudatus f. apsteini: Teeramaethee } \text { et al., } 2006 \\
\text { Brachionus caudatus f. personatus: Sanoamuang et al., } 1995\end{array}$ & $\mathrm{~N}, \mathrm{NE}, \mathrm{C}, \mathrm{S}$ & $1,3,4,5,7,9,17,20,21,22,24,26$ \\
\hline 20 & Brachionus dichotomus Shephard, 1911 & NE, C, S & $5,7,20,21,23,24$ \\
\hline 21 & $\begin{array}{l}\text { Brachionus dichotomus reductus Koste \& Shiel, } 1980 \\
\text { Syn.: B. dichotomus Shephard f. reductus Koste \& Shiel, 1980: } \\
\text { Sanoamuang et al., 1995; Sanoamuang, 1998; Sanoamuang et al., 2001; } \\
\text { Segers et al., 2004; Savatenalinton \& Segers, 2005; Teeramaethee } \text { et al., 2006; } \\
\text { Sanoamuang, 2007 }\end{array}$ & $\mathrm{N}, \mathrm{NE}$ & $4,9,13,17,18,22$ \\
\hline 22 & Brachionus diversicornis (Daday, 1883) & $\mathrm{N}, \mathrm{NE}, \mathrm{C}$ & $3,4,9,17,20,21,22,24,26$ \\
\hline 23 & Brachionus donneri Brehm, 1951 & $\mathrm{~N}, \mathrm{NE}, \mathrm{C}, \mathrm{S}$ & $3,4,5,7,9,13,17,20,22,23,24$ \\
\hline 24 & Brachionus durgae Dhanapathi, 1974 & $\mathrm{NE}$ & 4,24 \\
\hline 25 & Brachionus falcatus Zacharias, 1898 & $\mathrm{~N}, \mathrm{NE}, \mathrm{C}, \mathrm{S}$ & $\begin{array}{l}1,3,4,5,7,9,10,13,17,18,20,21,22,23,24, \\
26,28\end{array}$ \\
\hline 26 & $\begin{array}{l}\text { Brachionus forficula Wierzejski, } 1891 \\
\text { Incl. Brachionus forficula f. typica, f. reductus: Sanoamuang et al., } 1995\end{array}$ & $\mathrm{~N}, \mathrm{NE}, \mathrm{C}, \mathrm{S}$ & $\begin{array}{l}1,3,4,5,7,9,13,17,18,20,21,22,23,24,25, \\
26,28\end{array}$ \\
\hline 27 & Brachionus kostei Shiel, 1983 & $\mathrm{~N}, \mathrm{NE}$ & $4,9,17$ \\
\hline 28 & Brachionus leydigii Cohn, 1862 & - & 3 \\
\hline 29 & Brachionus lyratus Shephard, 1911 & $\mathrm{NE}, \mathrm{S}$ & $4,17,23,24$ \\
\hline 30 & $\begin{array}{l}\text { Brachionus murphyi Sudzuki, } 1989 \\
\text { Syn.: Brachionus niwati Sanoamuang, Segers \& Dumont, 1995: } \\
\text { Sanoamuang et al., 1995; Pholpunthin, 1997; Segers \& Pholpunthin, } 1997\end{array}$ & NE, S & $4,5,7,17,23$ \\
\hline 31 & Brachionus nilsoni Ahlstrom, 1940 & $\mathrm{C}$ & 22 \\
\hline 32 & Brachionus plicatilis Müller, 1786 & $\mathrm{NE}$ & 3,4 \\
\hline 33 & $\begin{array}{l}\text { Brachionus quadridentatus Hermann, } 1783 \\
\text { Syn.: Brachionus quadridentatus quadridentatus Hermann, 1783: }\end{array}$ & N, NE, C, S & $\begin{array}{l}1,3,4,5,7,9,10,13,15,17,18,20,21,22,23, \\
24,26\end{array}$ \\
\hline
\end{tabular}

Sanoamuang et al., 1995; Athibai et al., 2005

Brachionus quadridentatus f. typica: Sanoamuang et al., 1995

Incl. Brachionus quadridentatus f. brevispinus: Sanoamuang et al., 1995;

Athibai et al., 2005

Brachionus quadridentatus f. cluniorbicularis: Athibai et al., 2005

34 Brachionus quadridentatus melhemi Barrois \& Daday, 1894

Syn.: B. quadridentatus f. melheni Barrois \& Daday, 1894: Athibai et al., 2005

35 Brachionus quadridentatus mirabilis Daday, 1897

Syn.: B. quadridentatus f. mirabilis Daday, 1897:

Chittapun \& Pholpunthin, 2001; Chittapun et al., 2002; Athibai et al., 2005

Brachionus rotundiformis Tschugunoff, 1921

Brachionus rubens Ehrenberg, 1838

Brachionus sericus Rousselet, 1907

Brachionus sessilis Varga, 1951

Brachionus srisumonae Segers, Kothetip \& Sanoamuang, 2004

Brachionus urceolaris Müller, 1773

Brachionus variabilis Hempel, 1896

Keratella cochlearis (Gosse, 1851)

Incl. Keratella cochlearis f. typica, f. micracantha: Sanoamuang et al., 1995

Keratella edmondsoni Ahlstrom, 1943

Keratella javana Hauer, 1973

Keratella lenzi Hauer, 1953

Keratella mixta (Oparina-Charitonova, 1924)

Keratella procurva (Thorpe, 1891)

Keratella taksinensis Chittapun, Pholpunthin \& Segers, 2002

Keratella tecta (Gosse, 1851)

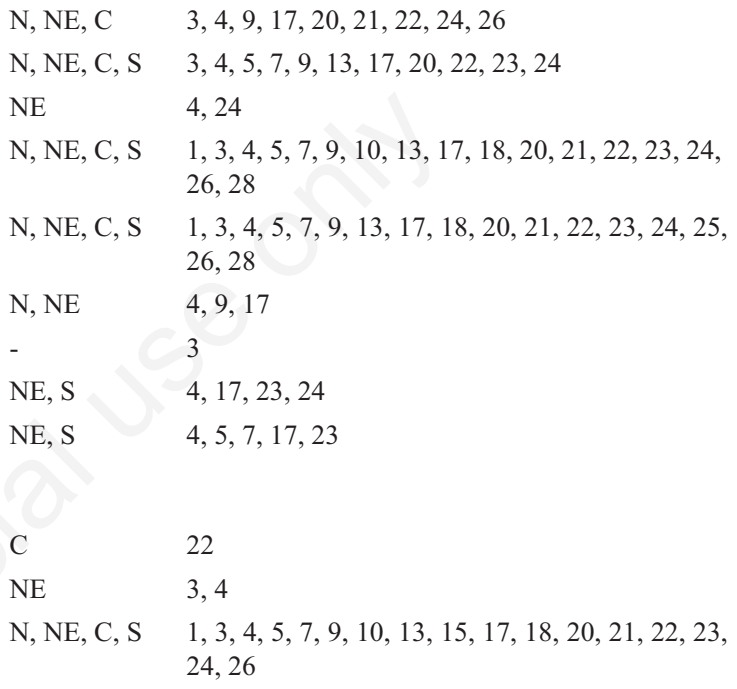

18

S $\quad 12,18$

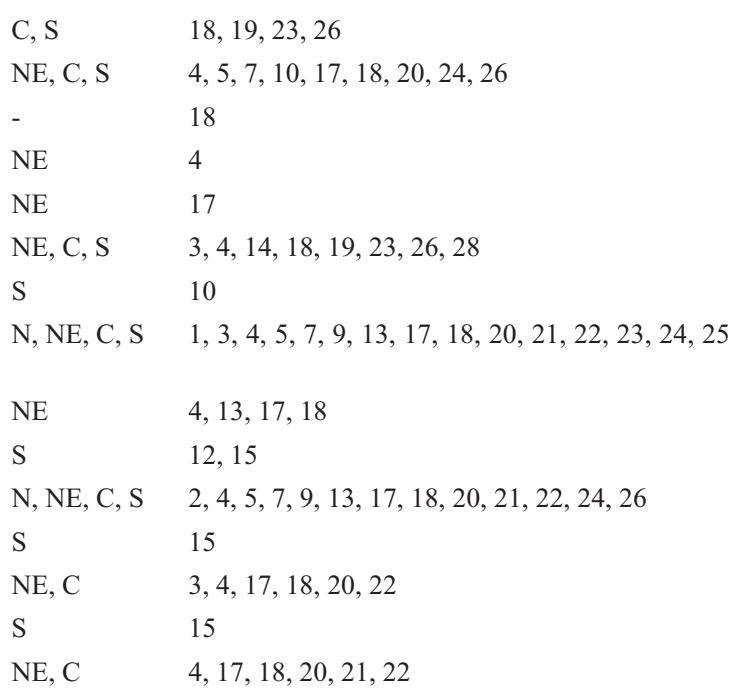

To be continued on next page. 
Tab. 1. Continued from previous page.

\begin{tabular}{|c|c|c|c|}
\hline & Species & Distribution & References \\
\hline 51 & $\begin{array}{l}\text { Keratella tropica (Apstein, 1907) } \\
\text { Syn.: Keratella valga tropica Apstein, 1907: Koste, } 1975\end{array}$ & $\mathrm{~N}, \mathrm{NE}, \mathrm{C}, \mathrm{S}$ & $\begin{array}{l}1,2,3,4,5,7,9,10,13,17,18,20,21,22,23, \\
24,25,26\end{array}$ \\
\hline 52 & $\begin{array}{l}\text { Plationus patulus (Müller, 1786) } \\
\text { Syn: Brachionus patulus Müller, 1786: Boonsom, } 1984 \\
\text { Plationus patulus (Müller) f. typica: Sanoamuang et al., } 1995\end{array}$ & $\mathrm{~N}, \mathrm{NE}, \mathrm{C}, \mathrm{S}$ & $\begin{array}{l}3,4,5,7,9,10,12,13,15,17,18,20,21,22,23, \\
24,26\end{array}$ \\
\hline \multirow[t]{2}{*}{53} & $\begin{array}{l}\text { Platyias quadricornis (Ehrenberg, 1832) } \\
\text { Syn.: Platyias quadricornis quadricornis (Ehrenberg, 1832): } \\
\text { Chittapun et al., } 2002 \\
\text { Incl. Platyias quadricornis f. brevispinus: Chittapun et al., } 2002\end{array}$ & $\mathrm{~N}, \mathrm{NE}, \mathrm{C}, \mathrm{S}$ & $3,4,5,7,9,10,13,15,17,18,21,22,23,24,26$ \\
\hline & Family Conochilidae & & \\
\hline 54 & Conochilus (Conochiloides) coenobasis (Skorikov, 1914) & $\mathrm{NE}$ & $4,13,20,24$ \\
\hline 55 & Conochilus (Conochiloides) dossuarius Hudson, 1885 & $\mathrm{~N}, \mathrm{NE}$ & 4,9 \\
\hline 56 & Conochilus (Conochiloides) natans (Seligo, 1900) & $\mathrm{NE}, \mathrm{S}$ & $4,13,20,23,24$ \\
\hline 57 & Conochilus (Conochilus) hippocrepis (Schrank, 1803) & $\mathrm{NE}, \mathrm{S}$ & $4,20,24,29$ \\
\hline 58 & Conochilus (Conochilus) unicornis Rousselet, 1892 & $\mathrm{NE}$ & 3,13 \\
\hline & Family Collothecidae & & 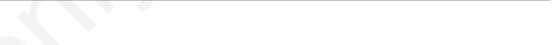 \\
\hline 59 & Collotheca algicola (Hudson, 1886) & $\mathrm{S}$ & 29 \\
\hline 60 & Collotheca ambigua (Hudson, 1883) & $\mathrm{S}$ & 29 \\
\hline 61 & $\begin{array}{l}\text { Collotheca campanulata (Dobie, 1849) } \\
\text { Incl. Collotheca campanulata var. longicaudata: Meksuwan et al., } 2011\end{array}$ & $\mathrm{NE}, \mathrm{S}$ & 4,29 \\
\hline 62 & Collotheca edentata (Collins, 1872) & $\mathrm{C}$ & 2 \\
\hline 63 & Collotheca heptabrachiata (Schoch, 1869) & $\mathrm{S}$ & 29 \\
\hline 64 & Collotheca ornata (Ehrenberg, 1832) & $\mathrm{S}$ & 29 \\
\hline 65 & Collotheca stephanochaeta Edmondson, 1936 & $\mathrm{~S}$ & 29 \\
\hline 66 & Collotheca tenuilobata (Anderson, 1889) & $\mathrm{NE}, \mathrm{S}$ & 13,29 \\
\hline 67 & $\begin{array}{l}\text { Collotheca trilobata (Collins, 1872) } \\
\text { Collotheca cf. trilobata (Collins, 1872): Teeramaethee et al., } 2006\end{array}$ & $\mathrm{~S}$ & 29 \\
\hline 68 & Stephanoceros fimbriatus (Goldfusz, 1820) & $\mathrm{S}$ & 29 \\
\hline 69 & Stephanoceros millsii (Kellicott, 1885) & $\mathrm{S}$ & 29 \\
\hline
\end{tabular}

Family Dicranophoridae

70 Aspelta circinator (Gosse, 1886)

NE $\quad 13$

71 Dicranophoroides caudatus (Ehrenberg, 1834)

Syn.: Dicranophorus caudatus (Ehrenberg, 1834): Sanoamuang and

$\mathrm{N}, \mathrm{NE} \quad 4,9,13,17,20$
Savatenalinton, 2001

72 Dicranophoroides claviger (Hauer, 1965) Syn.: Dicranophorus claviger (Hauer): Pholpunthin, 1997

73 Dicranophorus epicharis Harring \& Myers, 1928 Dicranophorus cf. epicharis Harring \& Myers, 1928: Sanoamuang et al., 1995

74 Dicranophorus grandis (Ehrenberg, 1832)

75 Dicranophorus prionacis Harring \& Myers, 1928

76 Dicranophorus robustus Harring \& Myers, 1928

77 Encentrum longidens Donner, 1943

78 Encentrum pornsilpi Segers \& Chittapun, 2001

\begin{tabular}{ll}
\hline & Family Epiphanidae \\
79 & Cyrtonia tuba (Ehrenberg, 1834) \\
80 & Epiphanes clavulata (Ehrenberg, 1832) \\
81 & Epiphanes macroura (Barrois \& Daday, 1894) \\
82 & Proalides subtilis Rodewald, 1940 \\
83 & Proalides tentaculatus de Beauchamp, 1907 \\
\hline
\end{tabular}
Family Euchlanidae

84 Beauchampiella eudactylota (Gosse, 1886) Syn.: Manfredium eudactylotum (Gosse, 1886):

$\begin{array}{ll}\text { NE, S } & 4,5,7,13,17,24 \\ \text { NE, C, S } & 2,10,12,13,17,20,23,26 \\ \text { N, NE } & 4,9,13 \\ \text { S } & 5,7 \\ - & 3 \\ \text { S } & 14 \\ \text { S } & 14,19,23\end{array}$

Sanoamuang et al., 1995; Sanoamuang, 1998; Chittapun et al., 2002; Segers et al., 2004 
Tab. 1. Continued from previous page.

\begin{tabular}{|c|c|c|c|}
\hline & Species & Distribution & References \\
\hline 85 & $\begin{array}{l}\text { Dipleuchlanis propatula (Gosse, 1886) } \\
\text { Incl. Dipleuchlanis propatula f. macrodactyla: Chittapun et al., } 2002\end{array}$ & $\mathrm{~N}, \mathrm{NE}, \mathrm{C}, \mathrm{S}$ & $3,4,5,7,9,12,13,15,17,20,23,24,26$ \\
\hline 86 & Euchlanis dilatata Ehrenberg, 1832 & $\mathrm{~N}, \mathrm{NE}, \mathrm{C}, \mathrm{S}$ & $1,2,3,4,5,7,9,10,12,13,17,20,22,23,24,26$ \\
\hline 87 & $\begin{array}{l}\text { Euchlanis incisa Carlin, } 1939 \\
\text { Incl. Euchlanis incisa f. mucronata: Chittapun et al., } 1999\end{array}$ & $\mathrm{~N}, \mathrm{NE}, \mathrm{C}, \mathrm{S}$ & $4,9,10,12,13,15,17,20,22,24$ \\
\hline 88 & $\begin{array}{l}\text { Euchlanis lyra Hudson, } 1886 \\
\text { Incl. Euchlanis lyra f. myersi: Chittapun et al., } 1999\end{array}$ & $\mathrm{~S}$ & 10 \\
\hline 89 & Euchlanis meneta Myers, 1930 & $\mathrm{NE}, \mathrm{C}, \mathrm{S}$ & $2,10,13$ \\
\hline 90 & Euchlanis triquetra Ehrenberg, 1838 & $\mathrm{C}$ & 2 \\
\hline 91 & Tripleuchlanis plicata (Levander, 1894) & $\mathrm{N}, \mathrm{NE}, \mathrm{C}$ & $1,3,4,9,13,17,21,22$ \\
\hline
\end{tabular}

Family Flosculariidae

92 Beauchampia crucigera (Dutrochet, 1812)

Syn.: Beauchampia crucigera crucigera (Dutrochet, 1812): Koste, 1975

93 Floscularia armata Segers, 1997

94 Floscularia bifida Segers, 1997

95 Floscularia conifera (Hudson, 1886)

96 Floscularia decora Edmondson, 1940

97 Floscularia melicerta (Ehrenberg, 1832)

98 Floscularia pedunculata (Joliet, 1883)

99 Floscularia ringens (Linnaeus, 1758)

100 Floscularia wallacei Segers \& Shiel, 2008

101 Lacinularia flosculosa (Müller, 1773)

102 Lacinularoides coloniensis (Colledge, 1918)

103 Limnias ceratophylli Schrank, 1803

104 Limnias melicerta Weisse, 1848

105 Octotrocha speciosa Thorpe, 1893

106 Pentatrocha gigantea Segers \& Shiel, 2008

107 Ptygura agassizi Edmondson, 1948

108 Ptygura barbata Edmondson, 1939

109 Ptygura beauchampi Edmondson, 1940

110 Ptygura brachiata (Hudson, 1886)

111 Ptygura crystallina (Ehrenberg, 1834)

112 Ptygura ctenoida Koste \& Tobias, 1990

113 Ptygura elsteri Koste, 1972

Syn.: Ptygura elsteri elsteri Koste, 1972: Koste, 1975

114 Ptygura furcillata (Kellicott, 1889)

Syn.: Ptygura furcillata furcillata (Kellicott, 1889): Koste, 1975

Incl.: Ptygura furcillata $\mathrm{f}$. variabilis: Koste, 1975

115 Ptygura kostei José de Paggi, 1996

Syn.: Ptygura elsteri f. thailandis Koste, 1975: Sanoamuang and Savatenalinton, 2001

116 Ptygura longicornis (Davis, 1867)

117 Ptygura melicerta Ehrenberg, 1832

118 Ptygura mucicola (Kellicott, 1888)

119 Ptygura noodti (Koste, 1972)

120 Ptygura pedunculata Edmondson, 1939

121 Ptygura tacita Edmondson, 1940

122 Ptygura thalenoiensis Meksuwan, Pholpunthin \& Segers, 2011

123 Ptygura wilsonii (Anderson \& Shephard, 1892)

124 Sinantherina ariprepres Edmondson, 1939

125 Sinantherina semibullata (Thorpe, 1893)

126 Sinantherina socialis (Linnaeus, 1758)

127 Sinantherina spinosa (Thorpe, 1893)

\begin{tabular}{|c|c|}
\hline $\mathrm{C}, \mathrm{S}$ & 2,29 \\
\hline $\mathrm{S}$ & 29 \\
\hline $\mathrm{S}$ & 29 \\
\hline $\mathrm{NE}, \mathrm{C}, \mathrm{S}$ & $2,12,13,17,19,23,29$ \\
\hline $\mathrm{C}$ & 2 \\
\hline $\mathrm{C}$ & 2 \\
\hline $\mathrm{S}$ & 29 \\
\hline $\mathrm{NE}, \mathrm{C}, \mathrm{S}$ & $2,13,29$ \\
\hline $\mathrm{S}$ & 29 \\
\hline $\mathrm{S}$ & 29 \\
\hline S & 29 \\
\hline $\mathrm{C}, \mathrm{S}$ & 2,29 \\
\hline $\mathrm{C}, \mathrm{S}$ & $2,14,29$ \\
\hline $\mathrm{S}$ & 29 \\
\hline $\mathrm{S}$ & 29 \\
\hline $\mathrm{S}$ & 29 \\
\hline $\mathrm{S}$ & 29 \\
\hline $\mathrm{S}$ & 29 \\
\hline $\mathrm{C}$ & 2 \\
\hline $\mathrm{S}$ & 29 \\
\hline $\mathrm{S}$ & 29 \\
\hline $\mathrm{C}, \mathrm{S}$ & 2,29 \\
\hline $\mathrm{NE}, \mathrm{C}, \mathrm{S}$ & $2,13,29$ \\
\hline
\end{tabular}

NE $\quad 13$

S 29

NE, C 2, 13

C, S 2, 29

S 29

S 29

$\mathrm{NE}, \mathrm{S} \quad 13,29$

S 29

S 29

NE, C $\quad 13,20,22$

$\mathrm{N}, \mathrm{NE}, \mathrm{S} \quad 4,9,20,29$

C, $\mathrm{S} \quad 2,29$

NE, C, S $\quad 4,13,17,20,22,24,26,29$

To be continued on next page. 
Tab. 1. Continued from previous page.

\begin{tabular}{|c|c|c|c|}
\hline & Species & Distribution & References \\
\hline \multicolumn{4}{|c|}{ Family Gastropodidae } \\
\hline 128 & Ascomorpha agilis Zacharias, 1893 & $\mathrm{C}$ & 21 \\
\hline 129 & Ascomorpha ecaudis Perty, 1850 & $\mathrm{~N}, \mathrm{NE}, \mathrm{C}, \mathrm{S}$ & $4,5,7,9,20,21$ \\
\hline 130 & Ascomorpha ovalis (Bergendal, 1892) & $\mathrm{NE}, \mathrm{C}, \mathrm{S}$ & $3,4,7,13,17,20,21,22,23,24$ \\
\hline 131 & Ascomorpha saltans Bartsch, 1870 & $\mathrm{NE}, \mathrm{C}, \mathrm{S}$ & $3,4,5,7,13,17,20,21,22$ \\
\hline 132 & Gastropus hyptopus (Ehrenberg, 1838) & $\mathrm{NE}$ & 4 \\
\hline \multicolumn{4}{|c|}{ Family Hexarthridae } \\
\hline 133 & Hexarthra fennica (Levander, 1892) & $\mathrm{NE}$ & 4 \\
\hline 134 & Hexarthra intermedia (Wiszniewski, 1929) & $\mathrm{N}, \mathrm{NE}, \mathrm{C}, \mathrm{S}$ & $4,5,7,9,13,17,20,21,22,24$ \\
\hline 135 & Hexarthra mira (Hudson, 1871) & $\mathrm{N}, \mathrm{NE}, \mathrm{C}, \mathrm{S}$ & $4,5,7,9,13,17,19,20,22,23,24$ \\
\hline 136 & Hexarthra oxyuris (Sernov, 1903) & $\mathrm{NE}$ & 4 \\
\hline \multicolumn{4}{|c|}{ Family Ituridae } \\
\hline 137 & Itura aurita (Ehrenberg, 1830) & $\mathrm{NE}$ & 4 \\
\hline 138 & Itura symmetrica Segers, Mbogo \& Dumont, 1994 & $\mathrm{NE}$ & 4 \\
\hline \multicolumn{4}{|c|}{ Family Lecanidae } \\
\hline 139 & Lecane abanica Segers, 1994 & $\mathrm{~S}$ & $12,23,27$ \\
\hline 140 & Lecane acanthinula (Hauer, 1938) & $\mathrm{NE}, \mathrm{S}$ & $4,6,14,23,27$ \\
\hline 141 & Lecane aculeata (Jakubski, 1912) & $\mathrm{N}, \mathrm{NE}, \mathrm{C}, \mathrm{S}$ & $\begin{array}{l}2,3,4,5,7,8,9,10,12,13,15,17,20,21,22, \\
23,24,27,28\end{array}$ \\
\hline 142 & Lecane acus (Harring, 1913) & $\mathrm{C}$ & 1,3 \\
\hline 143 & Lecane aeganea Harring, 1914 & $\mathrm{~N}, \mathrm{NE}, \mathrm{C}$ & $3,4,9,21,27$ \\
\hline 144 & Lecane agilis (Bryce, 1892) & $\mathrm{C}$ & 25,27 \\
\hline 145 & Lecane arcuata (Bryce, 1891) & $\mathrm{NE}, \mathrm{C}, \mathrm{S}$ & $1,2,3,4,10,12,25,27$ \\
\hline \multirow[t]{2}{*}{146} & Lecane arcula Harring, 1914 & & \\
\hline & Syn: Lecane strandi Berzins, 1943: Jithlang \& Wongrat, 2006 & $\mathrm{~N}, \mathrm{NE}, \mathrm{C}, \mathrm{S}$ & $4,5,7,8,9,10,13,15,17,20,22,23,24,27$ \\
\hline 147 & Lecane armata Thomasson, 1971 & - & 27 \\
\hline 148 & Lecane aspasia Myers, 1917 & $\mathrm{~N}, \mathrm{NE}$ & $4,9,17,27$ \\
\hline 149 & Lecane baimaii Sanoamuang \& Savatenalinton, 1999 & $\mathrm{NE}$ & $11,17,27$ \\
\hline 150 & Lecane batillifer (Murray, 1913) & $\mathrm{NE}, \mathrm{C}, \mathrm{S}$ & $4,5,7,17,20,22,23,27$ \\
\hline 151 & Lecane bifastigata Hauer, 1938 & $\mathrm{NE}, \mathrm{C}$ & $4,6,22,27$ \\
\hline 152 & Lecane bifurca (Bryce, 1892) & $\mathrm{NE}, \mathrm{S}$ & $5,7,10,19,20,23,24,27$ \\
\hline 153 & Lecane blachei Bērzinšš, 1973 & $\mathrm{~N}, \mathrm{NE}, \mathrm{C}, \mathrm{S}$ & $4,6,8,9,13,17,20,27$ \\
\hline 154 & Lecane braumi Koste, 1988 & $\mathrm{NE}, \mathrm{S}$ & $12,13,15,27$ \\
\hline 155 & $\begin{array}{l}\text { Lecane bulla (Gosse, 1851) } \\
\text { Syn.: Lecane bulla bulla (Gosse, 1851): Segers \& Savatenalinton, } 2010\end{array}$ & $\mathrm{~N}, \mathrm{NE}, \mathrm{C}, \mathrm{S}$ & $\begin{array}{l}1,2,3,4,5,7,8,9,10,12,13,14,15,17,19,20, \\
21,22,23,24,26,27,28\end{array}$ \\
\hline 156 & Lecane bulla diabolica (Hauer, 1936) & - & 27 \\
\hline 157 & Lecane calcaria Harring \& Myers, 1926 & $\mathrm{NE}, \mathrm{S}$ & $7,12,17,27$ \\
\hline 158 & Lecane chinesensis Zhuge \& Koste 1996 & - & 27 \\
\hline 159 & Lecane clara (Bryce, 1892) & NE, S & $5,7,12,20,27$ \\
\hline 160 & Lecane closterocerca (Schmarda, 1859) & $\mathrm{N}, \mathrm{NE}, \mathrm{C}, \mathrm{S}$ & $\begin{array}{l}1,2,3,4,5,7,9,10,12,13,15,17,20,21,22, \\
23,24,26,27\end{array}$ \\
\hline 161 & Lecane cornuta (Müller, 1786) & $\mathrm{C}$ & $1,2,3$ \\
\hline 162 & Lecane crenata (Harring, 1913) & $\mathrm{C}$ & 2,27 \\
\hline 163 & Lecane crepida Harring, 1914 & $\mathrm{~N}, \mathrm{NE}, \mathrm{C}, \mathrm{S}$ & $2,4,5,7,9,10,12,13,17,20,22,23,24,27$ \\
\hline 164 & $\begin{array}{l}\text { Lecane curvicornis (Murray, 1913) } \\
\text { Incl. Lecane curvicornis f. typica: Sanoamuang et al., } 1995\end{array}$ & $\mathrm{~N}, \mathrm{NE}, \mathrm{C}, \mathrm{S}$ & $\begin{array}{l}1,3,4,5,7,8,9,12,13,15,17,20,21,22,23, \\
24,26,27,28\end{array}$ \\
\hline 165 & Lecane decipiens (Murray, 1913) & $\mathrm{NE}, \mathrm{S}$ & $10,11,12,13,17,20,24,27$ \\
\hline 166 & Lecane donneri Chengalath \& Mulamoottil, 1974 & $\mathrm{NE}$ & $11,17,24,27$ \\
\hline 167 & Lecane doryssa Harring, 1914 & $\mathrm{~N}, \mathrm{NE}, \mathrm{S}$ & $4,9,12,13,15,17,20,23,24,27$ \\
\hline
\end{tabular}


Tab. 1. Continued from previous page.

\begin{tabular}{|c|c|c|c|}
\hline & Species & Distribution & References \\
\hline 168 & Lecane elegans Harring, 1914 & N, NE, C & $2,9,13,17,20,24,26,27,28$ \\
\hline 169 & Lecane enowi Segers and Mertens, 1997 & $\mathrm{~S}$ & 15,27 \\
\hline 170 & Lecane eswari Dhanapathi, 1976 & $\mathrm{NE}$ & $6,17,27$ \\
\hline 171 & Lecane flexilis (Gosse, 1886) & $\mathrm{NE}, \mathrm{C}, \mathrm{S}$ & $4,10,13,17,20,22,23,27$ \\
\hline 172 & Lecane furcata (Murray, 1913) & $\mathrm{N}, \mathrm{NE}, \mathrm{C}, \mathrm{S}$ & $\begin{array}{l}2,4,5,7,8,9,10,12,13,15,17,20,21,22,23, \\
24,27\end{array}$ \\
\hline 173 & $\begin{array}{l}\text { Lecane galeata (Bryce, 1892) } \\
\text { Syn.: Lecane (Monostyla) pygmaea Daday, 1897: Koste, } 1975\end{array}$ & $\mathrm{C}$ & 2 \\
\hline 174 & Lecane grandis (Murray, 1913) & $\mathrm{NE}, \mathrm{S}$ & $10,11,23,27$ \\
\hline 175 & Lecane haliclysta Harring \& Myers, 1926 & $\mathrm{~N}, \mathrm{NE}, \mathrm{C}, \mathrm{S}$ & $4,9,12,13,17,20,22,23,24,27$ \\
\hline 176 & Lecane hamata (Stokes, 1896) & $\mathrm{N}, \mathrm{NE}, \mathrm{C}, \mathrm{S}$ & $\begin{array}{l}2,4,5,7,8,9,10,12,13,14,15,17,20,21,22, \\
23,24,26,27\end{array}$ \\
\hline 177 & Lecane hastata (Murray, 1913) & $\mathrm{N}, \mathrm{NE}, \mathrm{C}, \mathrm{S}$ & $3,4,9,17,20,21,22,23,24,27$ \\
\hline 178 & Lecane hornemanni (Ehrenberg, 1834) & $\mathrm{N}, \mathrm{NE}, \mathrm{C}, \mathrm{S}$ & $\begin{array}{l}4,5,7,8,9,12,13,15,17,20,21,22,23,24, \\
26,27\end{array}$ \\
\hline 179 & Lecane inermis (Bryce, 1892) & $\mathrm{NE}, \mathrm{C}, \mathrm{S}$ & $2,4,5,7,10,12,15,19,20,23,26,27$ \\
\hline 180 & Lecane inopinata Harring \& Myers, 1926 & $\mathrm{~N}, \mathrm{NE}, \mathrm{C}, \mathrm{S}$ & $3,4,9,10,12,13,17,20,21,22,24,27,28$ \\
\hline 181 & Lecane intrasinuata (Olofsson, 1917) & $\mathrm{C}$ & 2 \\
\hline 182 & Lecane isanensis Sanoamuang \& Savatenalinton, 2001 & $\mathrm{NE}$ & 13,27 \\
\hline 183 & Lecane junki Koste, 1975 & $\mathrm{C}, \mathrm{S}$ & $2,6,16,27$ \\
\hline 184 & Lecane kunthuleensis Chittapun, Pholpunthin \& Segers, 2003 & $\mathrm{~S}$ & 16,27 \\
\hline 185 & Lecane lamellata (Daday, 1893) & - & 3 \\
\hline
\end{tabular}

186 Lecane lateralis Sharma, 1978

187 Lecane latissima Yamamoto, 1955

Syn.: Lecane thailandensis Segers \& Sanoamuang, 1994: Sanoamuang

N, NE, C, S $\quad 4,5,7,8,9,10,13,15,17,20,23,24,27,26,28$

$\mathrm{NE}, \mathrm{S} \quad 4,6,8,13,17,20,27$ et al., 1995; Sanoamuang and Segers, 1997 ; Pholpunthin \& Chittapun, 1998; Sanoamuang \& Savatenalinton, 2001; Segers et al., 2004; Savatenalinton \& Segers, 2005

188 Lecane lauterborni Hauer, 1924

189 Lecane leontina (Turner, 1892)

190 Lecane ludwigii (Eckstein, 1883)

191 Lecane luna (Müller, 1776)

192 Lecane lunaris (Ehrenberg, 1832)

193 Lecane lungae Savatenalinton \& Segers, 2005

194 Lecane martensi Savatenalinton \& Segers, 2008

195 Lecane micrognatha Segers \& Savatenalinton, 2010

196 Lecane minuta Segers, 1994

197 Lecane mitis Harring \& Myers, 1926

198 Lecane monostyla (Daday, 1897)

199 Lecane nana (Murray, 1913)

200 Lecane nelsoni Segers, 1994

201 Lecane nitida (Murray, 1913)

Syn.: Lecane curvicornis (Murray) f. nitida (Murray): Sanoamuang et al., 1995

202 Lecane niwati Segers, Kothetip \& Sanoamuang, 2004

203 Lecane obtusa (Murray, 1913)

204 Lecane opias (Harring \& Myers, 1926)

205 Lecane palinacis Harring \& Myers, 1926

206 Lecane papuana (Murray, 1913)

N, NE, C, S $2,4,5,7,8,9,10,12,13,15,17,20,21,22,23$, $24,26,27$

N, NE, C, S $\quad 2,4,5,7,8,9,10,12,13,15,17,19,20,22,23$, 24,27

N, NE, C, S $1,3,4,5,7,8,9,10,13,17,20,21,22,23,24$, $26,27,28$

N, NE, C, S $2,3,4,5,7,8,9,10,12,13,15,17,20,21,22$, $23,24,25,27$

NE $\quad 20,27$

C 25,27

- 27

S $\quad 5,6,7,27$

$\mathrm{S} \quad 12,27$

NE, C, S $\quad 3,4,10,12,15,17,22,23,24,27$

NE, C, S $\quad 1,3,4,5,7,13,17,20,27,28$

NE $\quad 13,27$

$\mathrm{NE} \quad 4,27$

NE $\quad 17,27$

$\mathrm{N}, \mathrm{NE}, \mathrm{C}, \mathrm{S} \quad 4,5,7,8,9,10,12,13,14,15,17,19,20,22,23$, 24,27

NE $\quad 20,27$

C, S $\quad 12,23,26,27,28$

N, NE, C, S 1, 3, 4, 5, 7, 8, 9, 10, 12, 13, 15, 17, 20, 21, 22, $24,26,27,28$

To be continued on next page. 
Tab. 1. Continued from previous page.

\begin{tabular}{|c|c|c|c|}
\hline & Species & Distribution & References \\
\hline 207 & Lecane paxiana Hauer, 1940 & $\mathrm{NE}, \mathrm{C}$ & $20,25,27$ \\
\hline 208 & Lecane pertica Harring \& Myers, 1926 & $\mathrm{~N}, \mathrm{NE}, \mathrm{S}$ & $5,7,9,12,13,15,17,27$ \\
\hline 209 & $\begin{array}{l}\text { Lecane punctata (Murray, 1913) } \\
\text { Syn.: Lecane harringi Ahlstrom, 1934: Boonsom, 1984; Jithlang and } \\
\text { Wongrat, } 2006\end{array}$ & $\mathrm{NE}, \mathrm{C}$ & $3,11,20,21,22,27$ \\
\hline 210 & Lecane pusilla Harring, 1914 & $\mathrm{~N}, \mathrm{NE}, \mathrm{S}$ & $4,9,12,13,17,20,27$ \\
\hline 211 & Lecane pyriformis (Daday, 1905) & $\mathrm{NE}, \mathrm{C}, \mathrm{S}$ & $2,4,10,12,13,14,15,17,20,22,23,24,26,27$ \\
\hline 212 & Lecane quadridentata (Ehrenberg, 1830) & $\mathrm{N}, \mathrm{NE}, \mathrm{C}, \mathrm{S}$ & $\begin{array}{l}1,2,3,4,5,7,8,9,10,12,13,17,22,23,24 \\
26,27\end{array}$ \\
\hline 213 & Lecane rhenana Hauer, 1929 & $\mathrm{~N}, \mathrm{NE}, \mathrm{C}, \mathrm{S}$ & $4,5,7,8,9,13,17,20,22,23,24,26,27$ \\
\hline 214 & Lecane rhytida Harring \& Myers, 1926 & $\mathrm{~N}, \mathrm{NE}, \mathrm{C}, \mathrm{S}$ & $5,7,9,10,12,14,17,22,23,27$ \\
\hline 215 & Lecane robertsonae Segers, 1993 & $\mathrm{NE}, \mathrm{S}$ & $17,20,23,27$ \\
\hline 216 & Lecane ruttneri Hauer, 1938 & $\mathrm{NE}$ & $4,13,17,20,24,27$ \\
\hline 217 & Lecane segersi Sanoamuang, 1996 & $\mathrm{NE}, \mathrm{C}, \mathrm{S}$ & $6,14,17,20,23,26,27$ \\
\hline 218 & Lecane serrata (Hauer, 1938) & $\mathrm{NE}$ & $11,17,27$ \\
\hline 219 & Lecane shieli Segers \& Sanoamuang, 1994 & $\mathrm{NE}, \mathrm{S}$ & $4,6,10,13,17,20,27$ \\
\hline 220 & Lecane signifera (Jennings, 1896) f. ploenensis (Voigt, 1902) & $\mathrm{N}, \mathrm{NE}, \mathrm{C}, \mathrm{S}$ & $\begin{array}{l}2,4,5,7,8,9,10,12,13,15,17,20,21,22,23 \\
24,26,27\end{array}$ \\
\hline 221 & Lecane simonneae Segers, 1993 & $\mathrm{NE}, \mathrm{S}$ & $12,13,15,17,27$ \\
\hline 222 & Lecane sola Hauer, 1936 & $\mathrm{NE}$ & $3,4,17,20,24,27$ \\
\hline 223 & Lecane stenroosi (Meissner, 1908) & $\mathrm{N}, \mathrm{NE}, \mathrm{C}, \mathrm{S}$ & $2,4,8,9,10,17,20,21,22,26,27$ \\
\hline 224 & Lecane stichaea Harring, 1913 & $\mathrm{C}$ & $2,21,27$ \\
\hline 225 & Lecane stichoclysta Segers, 1993 & $\mathrm{NE}$ & 20,27 \\
\hline 226 & Lecane subtilis Harring \& Myers, 1926 & $\mathrm{NE}, \mathrm{S}$ & $17,23,27$ \\
\hline 227 & Lecane superaculeata Sanoamuang \& Segers, 1997 & $\mathrm{~N}, \mathrm{NE}, \mathrm{C}, \mathrm{S}$ & $6,9,15,17,23,27$ \\
\hline 228 & Lecane sympoda Hauer, 1929 & $\mathrm{~S}$ & $5,7,27$ \\
\hline 229 & Lecane syngenes (Hauer, 1938) & $\mathrm{S}$ & 12,27 \\
\hline 230 & Lecane tenuiseta Harring, 1914 & $\mathrm{NE}, \mathrm{C}, \mathrm{S}$ & $\begin{array}{l}2,4,5,7,8,10,12,13,15,17,19,20,21,22,23, \\
24,27,28\end{array}$ \\
\hline 231 & Lecane thalera (Harring \& Myers, 1926) & $\mathrm{NE}, \mathrm{C}$ & $4,22,27$ \\
\hline 232 & Lecane thienemanni (Hauer, 1938) & $\mathrm{NE}, \mathrm{C}, \mathrm{S}$ & $11,12,17,20,21,22,23,24,26,27$ \\
\hline 233 & Lecane undulata Hauer, 1938 & $\mathrm{~N}, \mathrm{NE}, \mathrm{C}, \mathrm{S}$ & $4,5,7,9,12,13,17,20,23,24,27,28$ \\
\hline 234 & Lecane unguitata (Fadeev, 1926) & $\mathrm{N}, \mathrm{NE}, \mathrm{C}, \mathrm{S}$ & $\begin{array}{l}4,5,7,8,9,10,12,13,15,17,19,20,22,23,24 \\
26,27\end{array}$ \\
\hline \multirow[t]{2}{*}{235} & Lecane ungulata (Gosse, 1887) & $\mathrm{N}, \mathrm{NE}, \mathrm{C}, \mathrm{S}$ & $\begin{array}{l}2,4,5,7,8,9,10,12,13,15,17,20,21,22,23, \\
24,26,27\end{array}$ \\
\hline & Family Lepadellidae & & \\
\hline 236 & Colurella adriatica Ehrenberg, 1831 & NE, C, S & $1,3,4,12,13,20,23,24,25$ \\
\hline 237 & Colurella colurus (Ehrenberg, 1830) & $\mathrm{N}, \mathrm{NE}, \mathrm{C}, \mathrm{S}$ & $2,4,9,10,12,15,23,26$ \\
\hline 238 & Colurella colurus compressa (Lucks, 1912) & $\mathrm{S}$ & 14 \\
\hline 239 & Colurella hindenburgi Steinecke, 1917 & $\mathrm{C}$ & 21 \\
\hline 240 & Colurella obtusa (Gosse, 1886) & $\mathrm{NE}, \mathrm{C}, \mathrm{S}$ & $2,4,5,7,10,12,13,14,15,17,20,21,23,24$ \\
\hline 241 & Colurella psammophila Segers \& Chittapun, 2001 & $\mathrm{~S}$ & 14,23 \\
\hline 242 & Colurella sanoamuangae Chittapun, Pholpunthin \& Segers, 1999 & $\mathrm{C}, \mathrm{S}$ & $10,14,23,26$ \\
\hline 243 & Colurella sulcata (Stenroos, 1898) & NE, S & $12,13,20,23$ \\
\hline 244 & Colurella tesselata (Glascott, 1893) & $\mathrm{S}$ & 12,23 \\
\hline 245 & Colurella uncinata (Müller, 1773) & $\mathrm{N}, \mathrm{NE}, \mathrm{C}, \mathrm{S}$ & $5,7,9,12,13,15,17,20,22,23,24,26$ \\
\hline 246 & $\begin{array}{l}\text { Colurella uncinata bicuspidata (Ehrenberg, 1832) } \\
\text { Syn.: Colurella uncinata (Müller) f. bicuspidata (Ehrenberg): } \\
\text { Sanoamuang et al., 1995; Chittapun et al., } 2002\end{array}$ & $\mathrm{NE}$ & 4 \\
\hline 247 & Lepadella (Lepadella) acuminata (Ehrenberg, 1834) & $\mathrm{NE}, \mathrm{C}, \mathrm{S}$ & $1,3,4,10,13,17,20,21,23,24,26$ \\
\hline 248 & Lepadella (Lepadella) akrobeles Myers, 1934 & $\mathrm{NE}$ & 11,17 \\
\hline
\end{tabular}


Tab. 1. Continued from previous page.

\begin{tabular}{|c|c|c|c|}
\hline & Species & Distribution & References \\
\hline 249 & Lepadella (Lepadella) amphitropis Harring, 1916 & $\mathrm{NE}$ & 20 \\
\hline 250 & Lepadella (Lepadella) apsida Harring, 1916 & $\mathrm{NE}, \mathrm{S}$ & $4,5,7,10,13,23$ \\
\hline 251 & Lepadella (Lepadella) benjamini Harring, 1916 & $\mathrm{NE}$ & 13,17 \\
\hline 252 & Lepadella (Lepadella) biloba Hauer, 1958 & $\mathrm{NE}, \mathrm{S}$ & $4,5,7,13,17$ \\
\hline 253 & Lepadella (Lepadella) costatoides Segers, 1992 & $\mathrm{~N}, \mathrm{NE}, \mathrm{C}, \mathrm{S}$ & $4,9,13,17,20,22,23,24$ \\
\hline 254 & Lepadella (Lepadella) cristata (Rousselet, 1893) & $\mathrm{NE}, \mathrm{S}$ & 12,13 \\
\hline 255 & Lepadella (Lepadella) dactyliseta (Stenroos, 1898) & $\mathrm{NE}, \mathrm{S}$ & $4,5,7,12,24$ \\
\hline 256 & Lepadella (Lepadella) desmeti Segers \& Chittapun, 2001 & $\mathrm{~S}$ & 14,23 \\
\hline 257 & Lepadella (Lepadella) discoidea Segers, 1993 & $\mathrm{~N}, \mathrm{NE}, \mathrm{C}, \mathrm{S}$ & $4,9,10,12,13,15,17,22,24$ \\
\hline 258 & Lepadella (Lepadella) elliptica Wulfert, 1939 & $\mathrm{NE}$ & 3,20 \\
\hline 259 & $\begin{array}{l}\text { Lepadella (Lepadella) elongata Koste, } 1992 \\
\text { Lepadella cf. elongata Koste, 1992: Sanoamuang \& Savatenalinton, } 2001\end{array}$ & $\mathrm{NE}$ & $11,17,20$ \\
\hline 260 & Lepadella (Lepadella) eurysterna Myers, 1942 & $\mathrm{NE}, \mathrm{S}$ & $13,17,23$ \\
\hline 261 & Lepadella (Lepadella) latusinus (Hilgendorf, 1899) & $\mathrm{N}, \mathrm{NE}, \mathrm{C}, \mathrm{S}$ & $2,4,5,7,9,13,23$ \\
\hline 262 & Lepadella (Lepadella) lindaui Koste, 1981 & $\mathrm{NE}, \mathrm{C}, \mathrm{S}$ & $5,7,13,17,20,28$ \\
\hline 263 & Lepadella (Lepadella) minoruoides Koste \& Robertson, 1983 & $\mathrm{~S}$ & $5,7,23$ \\
\hline 264 & Lepadella (Lepadella) minuta (Weber \& Montet, 1918) & $\mathrm{C}$ & 25 \\
\hline 265 & Lepadella (Lepadella) ovalis (Müller, 1786) & $\mathrm{N}, \mathrm{NE}, \mathrm{C}, \mathrm{S}$ & $2,3,4,5,7,9,10,12,13,17,20,22,23,24,26$ \\
\hline 266 & Lepadella (Lepadella) patella (Müller, 1773) & $\mathrm{N}, \mathrm{NE}, \mathrm{C}, \mathrm{S}$ & $2,3,4,5,7,9,10,12,13,15,17,20,22,23,24,26$ \\
\hline 267 & Lepadella (Lepadella) punctata Wulfert, 1939 & $\mathrm{~S}$ & 16 \\
\hline 268 & Lepadella (Lepadella) quadricarinata (Stenroos, 1898) & N, NE, S & $4,5,7,9,10,12,13,17$ \\
\hline 269 & Lepadella (Lepadella) quinquecostata (Lucks, 1912) & $\mathrm{N}, \mathrm{NE}$ & $9,13,17$ \\
\hline 270 & Lepadella (Lepadella) rhomboides (Gosse, 1886) & $\mathrm{N}, \mathrm{NE}, \mathrm{C}, \mathrm{S}$ & $4,5,7,9,10,12,13,15,17,20,21,22,23,24,26$ \\
\hline 271 & Lepadella (Lepadella) triba Myers, 1934 & $\mathrm{NE}, \mathrm{C}, \mathrm{S}$ & $2,5,7,10,13,17,20,23,24$ \\
\hline 272 & $\begin{array}{l}\text { Lepadella (Lepadella) triptera (Ehrenberg, 1832) } \\
\text { Incl.: Lepadella triptera f. alata; Sanoamuang and Savatenalinton, 2001; } \\
\text { Segers, } 2004\end{array}$ & $\mathrm{NE}, \mathrm{S}$ & $4,13,17,20,23$ \\
\hline 273 & Lepadella (Lepadella) vandenbrandei Gillard, 1952 & N, NE, S & $4,5,7,9,12,13,15,17,20,23,24$ \\
\hline 274 & Lepadella (Heterolepadella) apsicora Myers, 1834 & $\mathrm{NE}, \mathrm{S}$ & $4,5,7,13,15,17,20,23,24$ \\
\hline 275 & Lepadella (Heterolepadella) ehrenbergii (Perty, 1850) & $\mathrm{N}, \mathrm{NE}, \mathrm{C}, \mathrm{S}$ & $4,9,13,15,17,20,22,23$ \\
\hline 276 & Lepadella (Heterolepadella) heterostyla (Murray, 1913) & $\mathrm{NE}, \mathrm{S}$ & $5,7,17,20$ \\
\hline 277 & $\begin{array}{l}\text { Lepadella (Xenolepadella) monodactyla Berzins, } 1960 \\
\text { Syn.: Lepadella monostyla f. caudata (Koste, 1972) }\end{array}$ & NE, C, S & $2,13,15,23$ \\
\hline 278 & Paracolurella aemula (Myers, 1934) & $\mathrm{S}$ & 16 \\
\hline 279 & $\begin{array}{l}\text { Squatinella lamellaris (Müller, 1786) } \\
\text { Syn.: Squatinella mutica (Ehrenberg): Chittapun \& Pholpunthin, 2001; } \\
\text { Chittapun et al., } 2007 \\
\text { Syn.: Squatinella lamellaris (Müller) f. mutica (Ehrenberg): Sanoamuang et al., } \\
\text { 1995; Sanoamuang \& Savatenalinton, 2001; Segers et al., } 2004\end{array}$ & $\mathrm{NE}, \mathrm{S}$ & $4,5,7,13,17,23$ \\
\hline 280 & $\begin{array}{l}\text { Squatinella leydigii (Zacharias, 1886) } \\
\text { Incl.: Squatinella leydigii f. longiseta: Chittapun et al., 1999; } \\
\text { Chittapun } \text { et al., } 2002\end{array}$ & $\mathrm{~S}$ & 10,15 \\
\hline
\end{tabular}
Family Lindiidae

281 Lindia (Lindia) torulosa Dujardin, 1841 $\mathrm{S}$

Family Mytilinidae

282 Lophocharis salpina (Ehrenberg, 1834)

283 Mytilina acanthophora Hauer, 1938

284 Mytilina bisulcata (Lucks, 1912)

285 Mytilina compressa (Gosse, 1851)

286 Mytilina crassipes (Lucks, 1912)

287 Mytilina michelangellii Reid \& Turner, 1988 Syn.: Mytilina ventralis f. diversicantha Wulfert, 1965: Jithlang \& Wongrat, 2006

$\begin{array}{ll}\text { N, NE, C } & 4,9,13,17,20,24,26 \\ \text { N, NE } & 4,9,13,17 \\ \text { N, NE, C } & 3,9,13,17,22,24,26 \\ \text { NE, S } & 4,5,7 \\ \text { C } & 21 \\ \text { C } & 21\end{array}$

To be continued on next page. 
Tab. 1. Continued from previous page.

\begin{tabular}{|c|c|c|c|}
\hline & Species & Distribution & References \\
\hline 288 & Mytilina mucronata (Müller, 1773) & $\mathrm{C}$ & 21 \\
\hline 289 & Mytilina unguipes (Lucks, 1912) & N, NE, C & $4,9,13,17,22,26$ \\
\hline 290 & Mytilina ventralis (Ehrenberg, 1830) & $\mathrm{N}, \mathrm{NE}, \mathrm{C}, \mathrm{S}$ & $3,4,9,10,12,13,17,20,21,22,23,24,26$ \\
\hline \multirow[t]{2}{*}{291} & Mytilina ventralis brevispina (Ehrenberg, 1830) & $\mathrm{C}$ & 21 \\
\hline & Family Notommatidae & & \\
\hline 292 & $\begin{array}{l}\text { Cephalodella forficula (Ehrenberg, 1830) } \\
\text { Syn.: Cephalodella forficula forficula (Ehrenberg, 1830): Koste, } 1975\end{array}$ & $\mathrm{NE}, \mathrm{C}, \mathrm{S}$ & $2,4,13,20,23,24$ \\
\hline 293 & Cephalodella gibba (Ehrenberg, 1830) & $\mathrm{NE}, \mathrm{C}, \mathrm{S}$ & $2,4,5,7,13,15,17,19,20,23,28$ \\
\hline 294 & Cephalodella cf. hyalina Myers, 1924 & $\mathrm{~S}$ & 10 \\
\hline 295 & Cephalodella innesi Myers, 1924 & $\mathrm{C}, \mathrm{S}$ & $10,14,15,19,23,28$ \\
\hline 296 & Cephalodella megalocephala (Glascott, 1893) & $\mathrm{S}$ & 14 \\
\hline 297 & Cephalodella mucronata Myers, 1924 & $\mathrm{NE}, \mathrm{C}, \mathrm{S}$ & $12,13,22$ \\
\hline 298 & Cephalodella cf. pachyodon Wulfert, 1937 & $\mathrm{NE}$ & 4 \\
\hline 299 & Cephalodella plicata Myers, 1924 & $\mathrm{~S}$ & 14 \\
\hline 300 & Cephalodella songkhlaensis Segers \& Pholpunthin, 1997 & $\mathrm{NE}, \mathrm{S}$ & 7,20 \\
\hline 301 & Cephalodella tenuior (Gosse, 1886) & $\mathrm{NE}, \mathrm{S}$ & 13,23 \\
\hline 302 & Cephalodella tenuiseta (Burn, 1890) & $\mathrm{C}$ & 28 \\
\hline 303 & Cephalodella ventripes (Dixon-Nuttall, 1901) & $\mathrm{NE}$ & 4 \\
\hline 304 & Eosphora cf. thoides Wulfert, 1935 & $\mathrm{NE}$ & 4 \\
\hline 305 & Monommata actices Myers, 1930 & $\mathrm{NE}, \mathrm{S}$ & $5,7,17$ \\
\hline 306 & Monommata dentata Wulfert, 1940 & $\mathrm{~S}$ & 15,23 \\
\hline 307 & Monommata grandis Tessin, 1890 & $\mathrm{~S}$ & $10,12,15,23$ \\
\hline 308 & Monommata longiseta (Müller, 1786) & $\mathrm{S}$ & $10,12,15$ \\
\hline 309 & Monommata maculata Harring \& Myers, 1930 & $\mathrm{~S}$ & 12 \\
\hline 310 & Notommata copeus Ehrenberg, 1834 & $\mathrm{NE}, \mathrm{S}$ & $4,5,7,13,23$ \\
\hline 311 & $\begin{array}{l}\text { Notommata pachyura (Gosse, 1886) } \\
\text { Incl.: Notommata pachyura f. spinosa: Chittapun \& Pholpunthin, } 2001\end{array}$ & N, NE, S & $4,9,12,13,17,20$ \\
\hline 312 & Notommata pseudocerberus de Beauchamp, 1908 & $\mathrm{~S}$ & 5,7 \\
\hline 313 & Notommata pygmaea Harring \& Myers, 1922 & $\mathrm{~S}$ & 10 \\
\hline 314 & Notommata saccigera Ehrenberg, 1830 & $\mathrm{~S}$ & $12,15,23$ \\
\hline \multirow[t]{2}{*}{315} & Taphrocampa annulosa Gosse, 1851 & $\mathrm{~S}$ & 10,23 \\
\hline & Family Scaridiidae & & \\
\hline 316 & Scaridium bostjani Daems \& Dumont, 1974 & $\mathrm{~N}, \mathrm{NE}, \mathrm{S}$ & $4,5,7,9,23$ \\
\hline 317 & Scaridium elegans Segers \& De Meester, 1994 & $\mathrm{NE}, \mathrm{S}$ & $11,12,13$ \\
\hline 318 & Scaridium grande Segers, 1995 & $\mathrm{~N}, \mathrm{NE}, \mathrm{S}$ & $9,12,13,15$ \\
\hline \multirow[t]{2}{*}{319} & Scaridium longicaudum (Müller, 1786) & $\mathrm{N}, \mathrm{NE}, \mathrm{C}, \mathrm{S}$ & $4,5,7,9,10,12,13,15,17,20,22,23,24$ \\
\hline & Family Synchaetidae & & \\
\hline 320 & Ploesoma lenticulare Herrick, 1885 & $\mathrm{NE}, \mathrm{C}$ & 13,22 \\
\hline 321 & Ploesoma hudsoni (Imhof, 1891) & $\mathrm{NE}, \mathrm{C}$ & $4,13,17,20,22$ \\
\hline 322 & Polyarthra euryptera Wierzejski, 1891 & - & 3 \\
\hline 323 & Polyarthra longiremis Carlin, 1943 & $\mathrm{NE}$ & 4 \\
\hline 324 & Polyarthra major Burckhardt, 1900 & $\mathrm{~N}, \mathrm{NE}$ & 4,9 \\
\hline 325 & Polyarthra minor Voigt, 1904 & $\mathrm{~S}$ & $5,7,10,15$ \\
\hline 326 & Polyarthra remata Skorikov, 1896 & $\mathrm{~S}$ & 5,7 \\
\hline 327 & $\begin{array}{l}\text { Polyarthra vulgaris Carlin, } 1943 \\
\text { Polyarthra cf. vulgaris Carlin, 1943: } \\
\text { Sanoamuang, } 2007\end{array}$ & $\mathrm{~N}, \mathrm{NE}, \mathrm{C}, \mathrm{S}$ & $1,3,4,5,7,9,10,12,13,17,20,21,22,23,26$ \\
\hline 328 & Synchaeta longipes Gosse, 1887 & $\mathrm{NE}$ & 4 \\
\hline 329 & Synchaeta pectinata Ehrenberg, 1832 & $\mathrm{~N}, \mathrm{NE}$ & $4,9,17$ \\
\hline 330 & Synchaeta stylata Wierzejski, 1893 & $\mathrm{NE}, \mathrm{C}$ & $4,13,17,20,21$ \\
\hline
\end{tabular}


Tab. 1. Continued from previous page.

\begin{tabular}{|c|c|c|c|}
\hline & Species & Distribution & References \\
\hline \multicolumn{4}{|c|}{ Family Tetrasiphonidae } \\
\hline 331 & Tetrasiphon hydrocora Ehrenberg, 1840 & $\mathrm{~S}$ & 12 \\
\hline \multicolumn{4}{|c|}{ Family Testudinellidae } \\
\hline 332 & Pompholyx complanata Gosse, 1851 & $\mathrm{~N}, \mathrm{NE}, \mathrm{C}$ & $1,3,4,9,17,20,21,22,24,26$ \\
\hline 333 & $\begin{array}{l}\text { Testudinella ahlstromi Hauer, } 1956 \\
\text { Syn.: Testudinella incisa ahlstromi (Hauer): Chittapun \& Pholpunthin, } 2001\end{array}$ & $\mathrm{~N}, \mathrm{NE}, \mathrm{S}$ & $9,12,13,17,20,24$ \\
\hline 334 & Testudinella amphora Hauer, 1938 & $\mathrm{NE}, \mathrm{S}$ & $12,13,17,23$ \\
\hline 335 & Testudinella brevicaudata Yamamoto, 1951 & $\mathrm{~N}, \mathrm{NE}, \mathrm{S}$ & $4,5,7,9,13,17,24$ \\
\hline 336 & Testudinella emarginula (Stenroos, 1898) & $\mathrm{NE}, \mathrm{S}$ & $4,17,23$ \\
\hline 337 & Testudinella greeni Koste, 1981 & $\mathrm{~N}, \mathrm{NE}$ & $9,13,17,24$ \\
\hline 338 & Testudinella mucronata (Gosse, 1886) & $\mathrm{S}$ & 12 \\
\hline 339 & $\begin{array}{l}\text { Testudinella parva (Ternetz, 1892) } \\
\text { Syn.: Testudinella insinuata Hauer, 1938: } \\
\text { Segers et al., 2004; T. cf. insinuata Hauer, 1938: Sanoamuang and } \\
\text { Savatenalinton, } 2001\end{array}$ & $\mathrm{NE}, \mathrm{C}, \mathrm{S}$ & $4,12,13,15,17,20,21,24$ \\
\hline 340 & $\begin{array}{l}\text { Testudinella patina }(\text { Hermann, 1783) } \\
\text { Syn.: Testudinella patina }(\text { Hermann) f. typica: Sanoamuang et al., } 1995 \\
\text { Incl.: Testudinella patina f. intermedia: Sanoamuang et al., 1995; Jithlang \& } \\
\text { Wongrat, } 2006\end{array}$ & $\mathrm{~N}, \mathrm{NE}, \mathrm{C}, \mathrm{S}$ & $\begin{array}{l}3,4,5,7,9,10,12,13,15,17,20,21,22,23, \\
24,26\end{array}$ \\
\hline 341 & Testudinella tridentata Smirnov, 1931 & $\mathrm{~N}, \mathrm{NE}, \mathrm{C}, \mathrm{S}$ & $4,9,12,13,15,17,20,24,26$ \\
\hline 342 & Testudinella walkeri Koste \& Shiel, 1980 & $\mathrm{NE}, \mathrm{S}$ & $5,7,13,17,20$ \\
\hline \multicolumn{4}{|c|}{ Family Trichocercidae } \\
\hline 343 & Trichocerca abilioi Segers \& Sarma, 1993 & NE & 13,17 \\
\hline 344 & Trichocerca bicristata (Gosse, 1887) & N, NE, C & $3,4,9,13,17,20,22,24$ \\
\hline 345 & Trichocerca bidens (Lucks, 1912) & NE, C, S & $4,13,17,20,22,23,24$ \\
\hline 346 & Trichocerca braziliensis (Murray, 1913) & $\mathrm{N}, \mathrm{NE}, \mathrm{C}, \mathrm{S}$ & $4,9,10,12,13,15,17,20,22,23,24,26$ \\
\hline 347 & Trichocerca capucina (Wierzejski \& Zacharias, 1893) & $\mathrm{N}, \mathrm{NE}, \mathrm{C}, \mathrm{S}$ & $4,9,13,17,20,21,22,23,24$ \\
\hline 348 & Trichocerca chattoni (de Beauchamp, 1907) & NE, C, S & $4,17,20,21,23,24$ \\
\hline 349 & Trichocerca collaris (Rousselet, 1896) & $\mathrm{NE}, \mathrm{S}$ & 12,13 \\
\hline 350 & Trichocerca cylindrica (Imhof, 1891) & NE, C, S & $3,4,5,7,13,17,20,21,22,24$ \\
\hline 351 & $\begin{array}{l}\text { Trichocerca dixonnuttalli (Jennings, 1903) } \\
\text { Syn.: Trichocerca inermis (Linder, 1904): Sanoamuang and Savatenalinton, } \\
\text { 2001; Sanoamuang } 2007\end{array}$ & $\mathrm{NE}, \mathrm{S}$ & $7,13,24$ \\
\hline 352 & Trichocerca elongata (Gosse, 1886) & $\mathrm{NE}, \mathrm{C}$ & $4,13,17,21$ \\
\hline 353 & Trichocerca flagellata Hauer, 1937 & $\mathrm{~N}, \mathrm{NE}, \mathrm{C}, \mathrm{S}$ & $4,5,7,9,12,13,17,22,23,24$ \\
\hline 354 & Trichocerca cf. gracilis (Tessin, 1890) & $\mathrm{S}$ & 23 \\
\hline 355 & Trichocerca hollaerti De Smet, 1990 & $\mathrm{NE}, \mathrm{S}$ & $4,5,7,12,13,17,23,24$ \\
\hline 356 & Trichocerca insignis (Herrick, 1885) & N, NE, S & $4,5,7,9,12,13,17,20,24$ \\
\hline 357 & $\begin{array}{l}\text { Trichocerca insulana (Hauer, 1937) } \\
\text { Syn.: Trichocerca montana Hauer, 1956: Sanoamuang \& Savatenalinton, } 2001\end{array}$ & NE, C, S & $4,10,13,20,23,24,26,28$ \\
\hline 358 & Trichocerca longiseta (Schrank, 1802) & $\mathrm{NE}, \mathrm{C}$ & $13,17,20,21,24$ \\
\hline 359 & Trichocerca mus Hauer, 1938 & $\mathrm{C}, \mathrm{S}$ & 22,23 \\
\hline 360 & Trichocerca orca (Murray, 1913) & $\mathrm{NE}$ & 13 \\
\hline 361 & Trichocerca porcellus (Gosse, 1851) & $\mathrm{NE}$ & $11,13,17$ \\
\hline 362 & Trichocerca pusilla (Jennings, 1903) & $\mathrm{N}, \mathrm{NE}, \mathrm{C}, \mathrm{S}$ & $1,3,4,9,10,13,17,19,20,21,22,23,24,25,26$ \\
\hline 363 & $\begin{array}{l}\text { Trichocerca obtusidens (Olofsson, 1918) } \\
\text { Syn.: Trichocerca relicta Donner, 1950: Sanoamuang et al., 1995; } \\
\text { Pholpunthin, 1997; Segers \& Pholpunthin, } 1997\end{array}$ & $\mathrm{NE}, \mathrm{S}$ & $4,5,7$ \\
\hline 364 & Trichocerca rosea (Stenroos, 1898) & $\mathrm{NE}$ & 13 \\
\hline 365 & Trichocerca rousseleti (Voigt, 1902) & $\mathrm{NE}$ & 4 \\
\hline 366 & Trichocerca ruttneri Donner, 1953 & NE, S & $4,5,7,13,23$ \\
\hline 367 & $\begin{array}{l}\text { Trichocerca scipio (Gosse, 1886) } \\
\text { Syn.: Trichocerca jenningsi Voigt, 1957: Sanoamuang \& Savatenalinton, 2001; }\end{array}$ & NE, S & $13,15,17$ \\
\hline
\end{tabular}

Syn.: Trichocerca jenningsi Voigt, 1957: Sanoamuang \& Savatenalinton, 2001; Chittapun et al., 2002 
Tab. 1. Continued from previous page.

\begin{tabular}{|c|c|c|c|}
\hline & Species & Distribution & References \\
\hline 368 & Trichocerca siamensis Segers \& Pholpunthin, 1997 & $\mathrm{NE}, \mathrm{S}$ & $7,13,15,20$ \\
\hline 369 & Trichocerca similis (Wierzejski, 1893) & $\mathrm{N}, \mathrm{NE}, \mathrm{C}, \mathrm{S}$ & $3,4,5,7,9,10,13,17,20,21,22,23,24$ \\
\hline 370 & $\begin{array}{l}\text { Trichocerca similis grandis Hauer, } 1965 \\
\text { Syn.: Trichocerca similis (Weirzejski) f. grandis Hauer: Chittapun \& } \\
\text { Pholpunthin, 2001; Chittapun et al., } 2002\end{array}$ & $\mathrm{C}, \mathrm{S}$ & $12,15,26$ \\
\hline 371 & Trichocerca simoneae De Smet 1990 & $\mathrm{NE}$ & $13,17,24$ \\
\hline 372 & Trichocerca stylata (Gosse, 1851) & NE, C & $4,13,17,22,24$ \\
\hline 373 & Trichocerca tenuidens (Hauer, 1931) & $\mathrm{NE}$ & 4 \\
\hline 374 & Trichocerca tenuior (Gosse, 1886) & $\mathrm{N}, \mathrm{NE}, \mathrm{S}$ & $3,9,13,14,17,19,20,23,24$ \\
\hline 375 & Trichocerca tigris (Müller, 1786) & $\mathrm{NE}, \mathrm{S}$ & $4,13,20,23$ \\
\hline 376 & $\begin{array}{l}\text { Trichocerca voluta (Murray, 1913) } \\
\text { Syn.: Trichocerca tropis Hauer, 1937: Sanoamuang et al., 1995; Pholpunthin, } \\
\text { 1997; Segers \& Pholpunthin, 1997; Chittapun \& Pholpunthin, 2001; } \\
\text { Sanoamuang \& Savatenalinton, 2001; Segers et al., } 2004\end{array}$ & $\mathrm{NE}, \mathrm{S}$ & $4,5,7,12,13,17$ \\
\hline 377 & Trichocerca vernalis (Hauer, 1936) & $\mathrm{NE}$ & 13 \\
\hline \multirow[t]{2}{*}{378} & Trichocerca weberi (Jennings, 1903) & $\mathrm{NE}, \mathrm{S}$ & $13,17,20,23$ \\
\hline & Family Trichotriidae & & \\
\hline 379 & Macrochaetus collinsii (Gosse, 1867) & $\mathrm{N}, \mathrm{NE}, \mathrm{C}, \mathrm{S}$ & $2,4,9,10,12,13,15,17,20,22,23,24$ \\
\hline 380 & Macrochaetus danneelae Koste \& Shiel, 1983 & $\mathrm{~N}, \mathrm{NE}$ & $9,13,17,24$ \\
\hline 381 & Macrochaetus longipes Myers, 1934 & $\mathrm{~N}, \mathrm{NE}, \mathrm{C}$ & $4,9,13,17,22,24$ \\
\hline 382 & Macrochaetus sericus (Thorpe, 1893) & $\mathrm{NE}, \mathrm{S}$ & $4,5,7,13,17,20,24$ \\
\hline 383 & Macrochaetus subquadratus (Perty, 1850) & $\mathrm{NE}, \mathrm{C}, \mathrm{S}$ & $12,13,17,22$ \\
\hline 384 & Trichotria tetractis (Ehrenberg, 1830) & $\mathrm{N}, \mathrm{NE}, \mathrm{C}, \mathrm{S}$ & $4,5,7,9,12,13,15,17,20,21,22,24$ \\
\hline 385 & Wolga spinifera (Western, 1894) & $\mathrm{NE}$ & $11,17,24$ \\
\hline & Family Trochosphaeridae & & \\
\hline 386 & Filinia brachiata (Rousselet, 1901) & $\mathrm{C}$ & 3,21 \\
\hline 387 & Filinia camasecla Myers, 1938 & $\mathrm{~N}, \mathrm{NE}, \mathrm{C}$ & $4,9,13,17,20,21,22,24,26,28$ \\
\hline 388 & $\begin{array}{l}\text { Filinia longiseta (Ehrenberg, 1834) } \\
\text { Incl.: Filinia longiseta var. limnetica: Jithlang \& Wongrat, } 2006\end{array}$ & $\mathrm{~N}, \mathrm{NE}, \mathrm{C}, \mathrm{S}$ & $1,3,4,9,10,17,20,21,22,23,24,26$ \\
\hline 389 & Filinia novaezealandiae Shiel \& Sanoamuang, 1993 & NE, C, S & $10,13,17,20,21,22,24,26,28$ \\
\hline 390 & $\begin{array}{l}\text { Filinia opoliensis (Zacharias, 1898) } \\
\text { Syn.: Tetramastix opoliensis Zacharias, 1898: De Ridder, } 1971\end{array}$ & $\mathrm{~N}, \mathrm{NE}, \mathrm{C}, \mathrm{S}$ & $1,3,4,9,10,12,13,17,20,21,22,23,24,26$ \\
\hline 391 & Filinia pejleri Hutchinson, 1964 & $\mathrm{~N}, \mathrm{NE}$ & 4,9 \\
\hline 392 & Filinia saltator (Gosse, 1886) & $\mathrm{N}, \mathrm{NE}$ & $4,9,17$ \\
\hline 393 & Filinia terminalis (Plate, 1886) & $\mathrm{NE}$ & 3,4 \\
\hline \multirow[t]{3}{*}{394} & Trochosphaera aequatorialis Semper, 1872 & $\mathrm{~N}, \mathrm{NE}, \mathrm{C}$ & $3,4,9,17,26$ \\
\hline & Bdelloidea & & \\
\hline & Family Habrotrochidae & & \\
\hline 395 & Habrotrocha ampulla Murray, 1911 & $\mathrm{C}$ & 2 \\
\hline 396 & Habrotrocha angusticollis (Murray, 1905) & $\mathrm{C}$ & 2 \\
\hline & Family Philodiniae & & \\
\hline 397 & Dissotrocha aculeata (Ehrenberg, 1832) & $\mathrm{C}, \mathrm{S}$ & $2,12,15,23$ \\
\hline 398 & Dissotrocha macrostyla (Ehrenberg, 1838) & $\mathrm{C}$ & 2 \\
\hline
\end{tabular}

$N$, northern; NE, northeastern; $C$, central; $S$, southern; -, no detail about region in publication; 1, De Ridder (1971); 2, Koste (1975); 3, Boonsom (1984); 4, Sanoamuang et al. (1995); 5, Pholpunthin (1997); 6, Sanoamuang and Segers (1997); 7, Segers and Pholpunthin (1997); 8, Pholpunthin and Chittapun (1998); 9, Sanoamuang (1998); 10, Chittapun et al. (1999); 11, Sanoamuang and Savatenalinton (1999); 12, Chittapun and Pholpunthin (2001); 13, Sanoamuang and Savatenalinton (2001); 14, Segers and Chittapun (2001); 15, Chittapun et al. (2002); 16, Chittapun et al. (2003); 17, Segers et al. (2004); 18, Athibai et al. (2005); 19, Chittapun et al. (2005); 20, Savatenalinton and Segers (2005); 21, Jithland and Wongrat (2006); 22, Teeramaethee et al. (2006); 23, Chittapun et al. (2007); 24, Sanoamuang (2007); 25, Savatenalinton and Segers (2008); 26, Chittapun et al. (2009); 27, Segers and Savatenalinton (2010); 28, Chittapun (2011); 29, Meksuwan et al. (2011). 
Tab. 2. List of Oriental and Thai taxa.

\begin{tabular}{|c|c|c|c|}
\hline & Oriental taxa & & Thai taxa \\
\hline 1 & Brachionus donneri Brehm, 1951 & 1 & Brachionus srisumonae Segers, Kothetip \& Sanoamuang, 2004 \\
\hline 2 & Brachionus murphyi Sudzuki, 1989 & 2 & Colurella psammophila Segers \& Chittapun, 2001 \\
\hline 3 & Cephalodella songkhlaensis Segers \& Pholpunthin, $1997^{*}$ & 3 & Encentrum pornsilpi Segers \& Chittapun, 2001 \\
\hline 4 & Colurella sanoamuangae Chittapun, Pholpunthin \& Segers, 1999 & 4 & Keratella taksinensis Chittapun, Pholpunthin \& Segers, 2002 \\
\hline 5 & Filinia camasecla Myers, 1938 & 5 & Lecane isanensis Sanoamuang \& Savatenalinton, 2001 \\
\hline 6 & Habrotrocha recumbens Bartoš, 1963 & 6 & Lecane junki Koste, 1975 \\
\hline 7 & Keratella edmondsoni Ahlstrom, 1943 & 7 & Lecane kunthuleensis Chittapun, Pholpunthin \& Segers, 2003 \\
\hline 8 & Lecane acanthinula (Hauer, 1938) & 8 & Lecane lungae Savatenalinton \& Segers, 2005 \\
\hline 9 & Lecane blachei Berzins, 1973 & 9 & Lecane martensi Savatenalinton \& Segers, 2008 \\
\hline 10 & Lecane bulla diabolica (Hauer, 1936) & 10 & Lecane micrognatha Segers \& Savatenalinton, 2010 \\
\hline 11 & Lecane minuta Segers, 1994 & 11 & Lecane niwati Segers, Kothetip \& Sanoamuang, 2004 \\
\hline 12 & Octotrocha speciosa Thorpe, 1893 & 12 & Lecane segersi Sanoamuang, 1996 \\
\hline 13 & Ptygura thalenoiensis Meksuwan, Pholpunthin \& Segers, 2011 & 13 & Lecane superaculeata Sanoamuang \& Segers, $1997^{*, o}$ \\
\hline
\end{tabular}

${ }^{*}$ Reliably recorded from Cambodia by Sor (2011); ' recorded, without substantiation, from Northeast India by Sharma and Sharma (2005).

\section{REFERENCES}

Athibai S, Sanoamuang L, Segers H, 2005. Species diversity of the family Brachionidae (Rotifera) in Thailand. KKU. Res. J. 5:1-10.

Balian EV, Segers H, Lévêque C, Martens K, 2008. An introduction to the Freshwater Animal Diversity Assessment (FADA) project. Hydrobiologia 595:3-8.

Boonsom J, 1984. The freshwater zooplankton of Thailand (Rotifera and Crustacea). Hydrobiologia 113:223-229.

Chittapun S, 2011. Fire and recovery of resting egg bank: an experimental study in paddy fields in Pathum Thani province, Thailand. Hydrobiologia 662:163-170.

Chittapun S, Pholpunthin P, 2001. The rotifer fauna of peat-swamps in southern Thailand. Hydrobiologia 446/447:226-269.

Chittapun S, Pholpunthin P, Segers H, 1999. Rotifera from peatswamps in Phuket Province, Thailand, with the description of a New Colurella Bory de St. Vincent. Int. Rev. Hydrobiol. 84:587-593.

Chittapun S, Pholpunthin P, Segers H, 2002. Rotifer diversity in a peat-swamp in southern Thailand (Narathiwas province) with the description of a new species of Keratella Bory de St. Vincent. Ann. Limnol.-Int. J. Limnol. 38:185-190.

Chittapun S, Pholpunthin P, Segers H, 2003. Contribution to the knowledge of Thai microfauna diversity: notes on rare peat swamp Rotifera, with the description of a new Lecane Nitzsch, 1872. Hydrobiologia 501:7-12.

Chittapun S, Pholpunthin P, Segers H, 2005. Restoration of tropical peat swamp rotifer communities after perturbation: an experimental study of recovery of rotifers from the resting egg bank. Hydrobiologia 546:281-289.

Chittapun S, Pholpunthin P, Segers H, 2007. Diversity of rotifer fauna from five coastal peat swamps on Phuket Island, Southern Thailand. ScienceAsia 33:383-387.

Chittapun S, Pholpunthin P, Sanoamuang L, 2009. Diversity and composition of zooplankton in rice fields during a crop cycle at Pathum Thani Province, Thailand. Songklanakarin J. Sci. Technol. 31:261-267.
Ciros-Pérez J, Gómez A, Serra M, 2001. On the taxonomy of three sympatric sibling species of the Brachionus plicatilis (Rotifera) complex from Spain, with the description of $B$. ibericus n. sp. J. Plankton Res. 23:1311-1328.

De Ridder M, 1971. [Raderdieren uit het verre Oosten]. [Article in Dutch]. Biol. Jb. Dodonaea 39:361-391.

Fontaneto D, Kaya M, Herniou EA, Barraclough TG, 2009. Extreme levels of hidden diversity in microscopic animals (Rotifera) revealed by DNA taxonomy. Mol. Phylogenet. Evol. 53:182-189.

García-Morales AE, Elías-Gutiérrez M, 2013. DNA barcoding of freshwater Rotifera in Mexico: evidence of cryptic speciation in common rotifers. Mol. Ecol. Resour. 13:1097-1107.

ICZN, 1999. International code of zoological nomenclature. The International Commission on Zoological Nomenclature ed., London: 306 pp.

Jersabek CD, De Smet WH, Fischer C, Fontaneto D, Michaloudi E, Wallace RL, Segers H, 2011. List of available names in zoology, part phylum Rotifera, species-group names established before 1 January 2000. Available from: http://www. ansp.org/ /media/Files/ans/research/sys-rotifera/LAN_CandidatePart-Rotifera-2012-03-22.ashx

Jithland I, Wongrat L, 2006. Composition and distribution of zooplankton in the Pakak Jolasid Reservoir, Lop Buri Province. KU. Fish. Res. Bull. 30:1-18.

Koste W, 1975. [Über den Rotatorienbestand einer Mikrobiozönose in einem tropischen aquatischen Saumbiotop, der Eichhornia-crassipes-Zone im Litoral des Bung-Borapet, einem Stausee in Zentralthailand]. [Article in German]. Gewässer Abwässer 57/58:43-58.

Leasi F, Tang CQ, De Smet WH, Fontaneto D, 2013. Cryptic diversity with wide salinity tolerance in the putative euryhaline Testudinella clypeata (Rotifera, Monogononta). Zool. J. Linn. Soc.-Lond. 168:17-28.

Meksuwan P, Pholpunthin P, Segers H, 2011. Diversity of sessile rotifers (Gnesiotrocha, Monogononta, Rotifera) in Thale Noi Lake, Thailand. Zootaxa 2997:1-18. 
Pholpunthin P, 1997. Freshwater zooplankton (Rotifera, Cladocera and Copepoda) from Thale-Noi, South Thailand. J. Sci. Soc. Thailand. 23:23-34.

Pholpunthin P, Chittapun S, 1998. Freshwater Rotifera of the genus Lecane from Songkhla Province, southern Thailand. Hydrobiologia 387/388:23-26.

Sanoamuang L, 1998. Rotifera of some freshwater habitats in the floodplain of the River Nan, northern Thailand. Hydrobiologia 387/388:27-33.

Sanoamuang L, 2007. The rotifer communities of temporary waters in Northeast Thailand. KKU Res. J. 12:203-209.

Sanoamuang L, Savatenalinton S, 1999. New records of rotifers from Nakhon Ratchasima province, northeast Thailand, with a description of Lecane baimaii n. sp. Hydrobiologia 412:95-101.

Sanoamuang L, Savatenalinton S, 2001. The rotifer fauna of Lake Kud-Thing, a shallow lake in Nong Khai Province, northeast Thailand. Hydrobiologia 446/447:297-304.

Sanoamuang L, Segers H, 1997. Additions to the Lecane Fauna (Rotifera: Monogononta) of Thailand. Int. Rev. Ges. Hydrobio. 82:525-530.

Sanoamuang L, Segers H, Dumont HJ, 1995. Additions to the rotifer fauna of south-east Asia: new and rare species from north-east Thailand. Hydrobiologia 313/314:35-45.

Savatenalinton S, Segers H, 2005. Rotifers from Kalasin Province, Northeast Thailand, with notes on new and rare species. Zool. Stud. 44:361-367.

Savatenalinton S, Segers H, 2008. Rotifer of waterfall mosses from Phu Hin Rong Kla National Park, Thailand, with the description of Lecane martensi, new species (Rotifera: Monogononta: Lecanidae). Raffles B. Zool. 56:245-249.

Segers H, 1996. The biogeography of littoral Lecane Rotifera. Hydrobiologia 323:169-197.

Segers H, 2001. Zoogeography of the southeast Asian Rotifera. Hydrobiologia 446/447:233-246.

Segers H, 2003. A biogeographical analysis of rotifers of the genus Trichocerca Lamarck, 1801 (Trichocercidae, Monogononta, Rotifera), with notes on taxonomy. Hydrobiologia 500:103-114.

Segers H, 2007. Annotated checklist of the rotifers (Phylum Rotifera), with notes on nomenclature, taxonomy and distribution. Zootaxa 1564:1-104.

Segers H, 2008. Global diversity of rotifers (Phylum Rotifera) in freshwater. Hydrobiologia 595:49-59.
Segers H, Chittapun S, 2001. The interstitial Rotifera of a tropical freshwater peat swamp on Phuket Island, Thailand. Belg. J. Zool. 131:65-71.

Segers H, De Smet WH, 2008. Diversity and endemism in Rotifera: a review, and Keratella Bory de St Vincent. Biodivers. Conserv. 17:303-316.

Segers H, Pholpunthin P, 1997. New and rare Rotifera from Thale-Noi Lake, Pattalang Province, Thailand, with a note on the taxonomy of Cephalodella (Notommatidae). Ann. Limnol.-Int. J. Limnol. 33:13-21.

Segers H, Savatenalinton S, 2010. A critical re-evaluation of the Lecanidae (Rotifera: Monogononta) of Thailand, with description of a new species. Int. Rev. Hydrobiol. 95:343-351.

Segers H, Koste W, Yussuf SM, 1996. Contribution to the knowledge of the monogonont Rotifera of Zanzibar, with a note on Filinia novaezealandiae Shiel \& Sanoamuang, 1993. Int. Rev. Ges. Hydrobio. 81:597-603.

Segers H, Kotethip W, Sanoamuang L, 2004. Biodiversity of freshwater microfauna in the floodplain of the River Mun, Northeast Thailand: the Rotifera Monogononta. Hydrobiologia 515:1-9.

Setapan Y, 1999. Geography of Thailand. 2. Pisit Publ., Bangkok: $457 \mathrm{pp}$

Sharma BK, Sharma S, 2005. Biodiversity of freshwater rotifers (Rotifera, Eurotatoria) from North-Eastern India. Zoosyst. Evol. 81:81-88.

Sor R, 2011. Comparison of the rotifer Fauna between lakes and reservoirs in the upper part of the Cambodian Mekong River Basin. Degree Thesis. Royal University of Phnom Phen ed., Phnom Phen: 75 pp.

Suatoni E, Vicario S, Rice S, Snell T, Caccone A, 2006. An analysis of species boundaries and biogeographic patterns in a cryptic species complex: the rotifer-Brachionus plicatilis. Mol. Phylogenet. Evol. 41:86-98.

Teeramaethee J, Trevanich A, Sanoamuang L, 2006. Biodiversity and abundance of rotifers in Bueng Boraphet, Nakhon Sawan Province, Thailand. KKU Res. J. 11:191-202.

Wallace RL, Snell TW, Ricci C, Nogrady T, 2006. Rotifera, 1: biology, ecology and systematics. In: H.J. Dumont, T. Nogrady, H. Segers (eds.), Guides to the identification of the microinvertebrates of the continental waters of the world. Kenobi Productions and Backhuys Publ.

Weber EF, 1907. [Rotateurs (Voyage du Dr. Walter Voltz)]. [Article in French]. Zool. Jahrb. Abt. System. 24:207-224. 